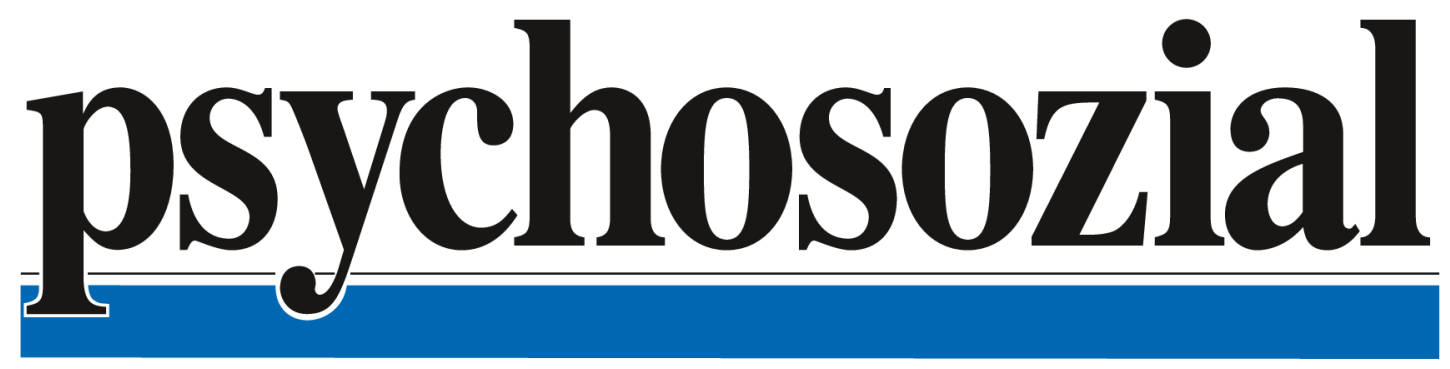

Herausgegeben von Michael B. Buchholz, Pradeep Chakkarath, Oliver Decker, Jörg Frommer, Benigna Gerisch, Rolf Haubl, Marie-Luise Hermann, Vera King, Carlos Kölbl, Joachim Küchenhoff, Jan Lohl, Katja Sabisch, Jürgen Straub und Hans-Jürgen Wirth

ISSN 0171-3434・42. Jahrgang • Nr. 158・2019・Heft IV
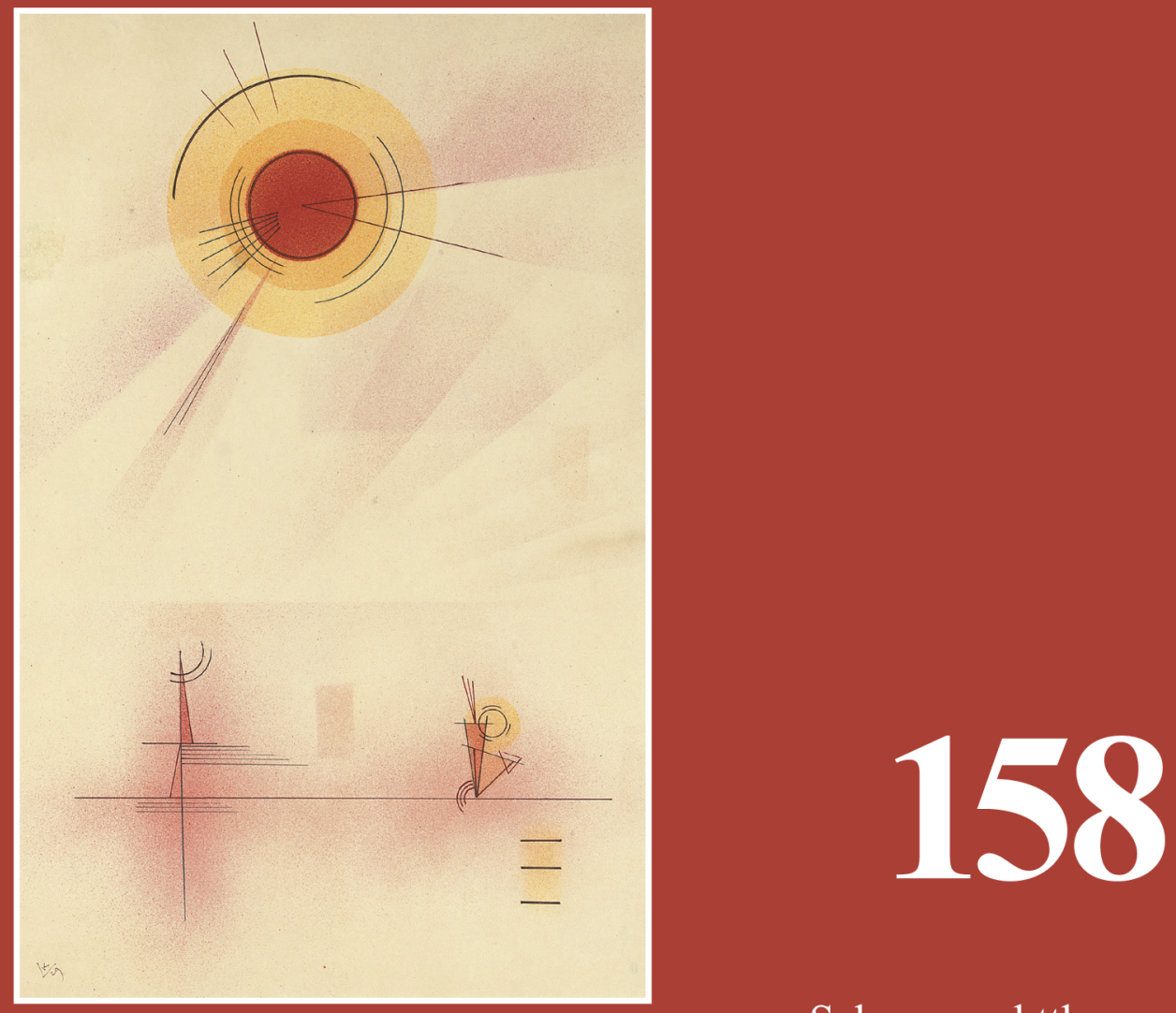

\title{
Diskurse der Achtsamkeit
}

Herausgegeben von Viktoria Niebel und Jürgen Straub 
https://doi.org/10.30820/0171-3434-2019-4-1

Generiert durch IP '172.22.53.54', am 26.04.2023, 13:09:55. 


\section{Inhalt}

\section{Schwerpunktthema:}

Diskurse der Achtsamkeit

Die allgegenwärtige Aktivierung

innerer Ressourcen

Diskurse der Achtsamkeit

in westlichen Gesellschaften

Viktoria Niebel \& Jürgen Straub

»Freiheit zur Leere

oder Freiheit zur Lehre?«

Achtsamkeit in der Schule

Cornelie Dietrich

\& Niels UhlendorF

Das Selbst kultivieren

Praktiken der Achtsamkeit in spirituellen und psychotherapeutischen Handlungsfeldern

Clemens Eisenmann

\& Frank Oberzaucher

(Um-)Deutungen subjektiver

Erfahrungen durch den Bezug auf »Achtsamkeit« im Kontext von Therapeutisierung

Simone RassmanN

»What you practice grows stronger «

Verkündungen transformatorischer

Potenziale von Achtsamkeit zwischen

neuroplastischen Gehirnen, Positiver

Psychologie und auteronomen Subjekten

VikToRia Niebel

Achtsamkeit, Intuition

und wissenschaftliche Erkenntnis

Eine ästhetisierende Perspektive

am Beispiel der qualitativen Sozialforschung

Maya Halatcheva-Trapp

\section{Freie Beiträge}

Psychoanalyse und Übersetzung -

Psychoanalyse der Übersetzung

ELLEN ReINKe
Antisemitismus

Eine genealogische Betrachtung

Bernd Auerochs

\section{Rezensionen}

Freuds Kokainstudien im Kontext

der zeitgenössischen Forschung

Anna Lindemann (2018). Sigmund Freud, das >Cocain < und die Morphinisten

18 Gerhard BenetKa

Perspektiven wissenschaftlicher Praxis

Pradeep Chakkarath \& Doris Weidemann (Hrsg.). (2018). Kulturpsychologische Ge-

31 genwartsdiagnosen: Bestandsaufnahmen $z u$ Wissenschaft und Gesellschaft

Charlotte Heidebrecht

Heilkunde für Menschen 4.0 -

Zurück in die Zukunft

Volker Roelcke (2017). Vom Menschen in der Medizin. Für eine kulturwissenschaftlich

kompetente Heilkunde

49 Robert E. Feldmann, JR.

Liebe und der Kampf

61 um soziale Emanzipation

Friedrich Voßkühler (2017). Ich - Du - Wir.

Liebe als zwischenmenschliche Wahrhaftig-

keit? Eine philosophische Erkundung in elf

Durchgängen

ERICH BAUER

Dank an die Gutachterinnen

76 und Gutachter

Impressum 


\section{Widersprüche \\ Zeitschrift für sozialistische Politik im Bildungs-, Gesundheits- und Sozialbereich}

Die Zeitschrift Widersprüche ist ein Forum für Theorie und Politik des Sozialen, Kritik und Kontroverse sowie für Entwürfe alternativer Praxis. Widersprüche informiert Sie viermal im Jahr kritisch, undogmatisch, theorieorientiert und praxisnah zu einem Schwerpunktthema.

www.widersprueche-zeitschrift.de

Heft 153

Die Macht von

Bezeichnungen

Zur Aktualität von

Etikettierungstheorien

2019 - ca. 130 Seiten - 15,00€

ISBN 978-3-89691-023-3

Heft 152

Trauma in Zeiten globaler

Selbstoptimierung

Heft 151

Kritische Solidaritäten?

Heft 150

200 Jahre Karl Marx -

das Alte im Neuen, das

Neue im Alten

Heft 149

Bestärken und Einsperren

Kindeswohl als

Kindeswohlgefährdung?

Die Widersprüche erscheinen im

Die Macht von Bezeichnungen Zur Aktualität von Etikettierungstheorien

Heidi Schulze \& Michael May

(Wie) Können Traumatisierte sprechen? Eine dialogische Suchbewegung

Roland Anhorn

Trauma, Traumatisierung, Posttraumatische Belastungsstörung: Vom (gescheiterten) Versuch einer emanzipatorischen Politisierung von sozialen Konflikten

Marcus Balzereit

Trauma-Politiken-Invers. Alternativen der Bearbeitung menschlichen Elends und dessen psychische Folgen, in der Perspektive kritischer internationaler Hilfs- und Menschenrechtsarbeit

Julia Manek

Trauma, Terror, Territorium - Interdisziplinäre Überlegungen zum kritischen Potential von Trauma-Konzepten

Ariane Brenssell

Das Forschungsprojekt "Kontextualisierte Traumaarbeit" Schlaglichter einer partizipativen Forschung

Eva Georg

Ambivalenzen eines hegemonialen Traumadiskurses im Kontext Beratung und Soziale Arbeit. Zwischen Einspruch und Anspruch

Forum

Felix Busch-Geertsema

Eine ausgebrannte Feuerwehr kann keine Brände löschen Selbstüberlastung in der Flüchtlingssozialarbeit

AKS Aachen

Einladung zum überregionalen Treffen der Arbeitskreise Kritische Soziale Arbeit
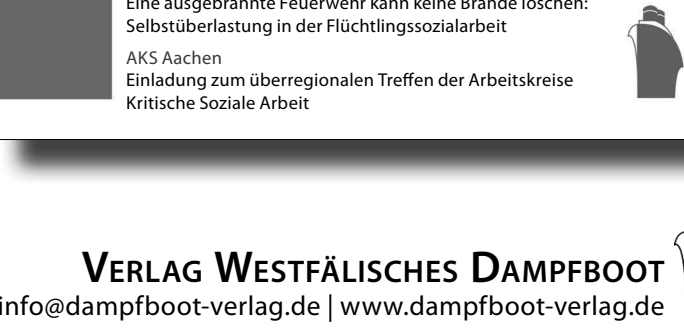
Schwerpunktthema:

\section{Diskurse der Achtsamkeit}

Herausgegeben von Viktoria Niebel und Jürgen Straub 


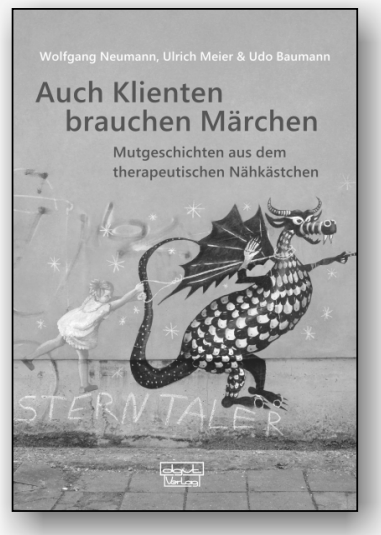

Wolfgang Neumann, Ulrich Meier \& Udo Baumann

\title{
Auch Klienten brauchen Märchen
}

\author{
2017, 152 Seiten \\ EUR 14,90 \\ ISBN 978-3-87159-267-6 \\ Auch als E-Book erhältlich: \\ ISBN 978-3-87159-422-9, EUR 12,99
}

Das Autorentrio vermittelt in seinem neuen praxisnahen Buch viele hilfreiche Tipps und Anregungen zum therapeutischen Umgang mit Sprache in der

Therapie. Es möchte Psychotherapeut*innen in Praxen und Beratungsstellen dazu ermutigen, den eigenen kreativen Impulsen zu ver-trauen. Zur Illustration werden unterschiedliche Beispiele aus der alltäglichen Praxis angeführt und dargestellt, wie mithilfe von Märchen und Geschichten auf „spielerische“ Weise Lösungen erreicht werden können.

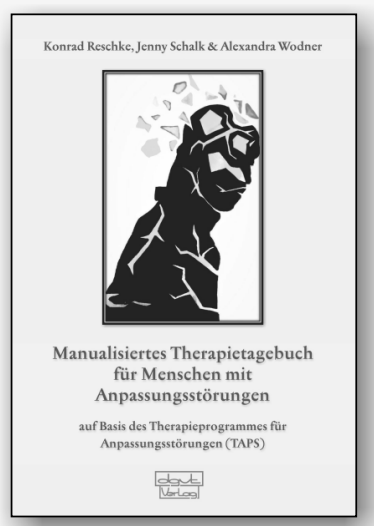

Konrad Reschke, Jenny Schalk \& Alexandra Wodner

\section{Manualisiertes Therapietagebuch für Menschen mit Anpassungsstörungen}

Auf Basis des Therapieprogrammes für Anpassungsstörungen (TAPS)

\author{
Materialie 71 \\ 2019, 188 Seiten \\ EUR 17,80 \\ ISBN 978-3-87159-371-0
}

Dieses Buch basiert auf den in der wissenschaftlichen Literatur veröffentlichten grundlegenden Erkenntnissen zu den verschiedenen Aspekten der Anpassungsstörung. Bei dem vorgestellten therapeutischen Programm handelt es sich um ein manualisiertes, kognitiv-behaviorales und theoriegeleitet störungsspezifisches Behandlungskonzept.

Das manualisierte Therapietagebuch für Menschen mit Anpassungsstörungen wurde als eine Art Tagebuch für die Praxis zur Unterstützung in der Einzelpsychotherapie entwickelt. Es enthält neben unterstützenden Fragen zur täglichen und wöchentlichen Selbstdokumentation zehn evidenzbasierte Interventionsbausteine.

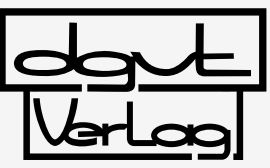

